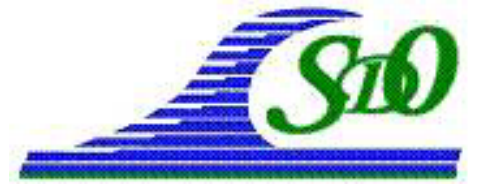

XI ${ }^{\text {èmes }}$ Journées Nationales Génie Côtier - Génie Civil

Les Sables d'Olonne, 22-25 juin 2010

DOI:10.5150/jngcgc.2010.044-M C Editions Paralia CFL

disponible en ligne - http://www.paralia.fr - available online

\title{
Morphodynamique de la plage de la Capte, Hyères, Var, suite à la mise en place d'atténuateurs de houle en géotextile
}

\author{
Samuel MEULÉ ${ }^{1}$, Anne-Éléonore PAQUIER ${ }^{1}$, Raphaël CERTAIN ${ }^{2}$, \\ Fréderic BOUCHETTE $^{3}$, Julien GRATIOT ${ }^{4}$, \\ François SABATIER ${ }^{1}$, Nicolas ROBIN ${ }^{2}$
}

1. Centre Européen de Recherche et d'Enseignement des Géosciences de

l'Environnement, Unité Mixte de Recherche 6635 CNRS/Université Aix-Marseille, Europôle de l'Arbois, BP 80, 13545 Aix-en-Provence Cedex 04, France. meule@cerege.fr

2. IMAGES-Université de Perpignan Via-Domitia, 52 Av Paul Alduy, 66860 Perpignan, France.

3. Institut des Sciences de la Terre, de l'Environnement et de l'Espace, FR 2035, Université de Montpellier II, F-34095 Montpellier, France.

4. Laboratoire Environnement Marin Littoral, EA4228 ECOMERS, Université de NiceSophia Antipolis (UNSA), Faculté des Sciences, Parc Valrose, 06108 Nice cedex 2, France.

\section{Résumé :}

La Ville de Hyères dans le Var procède chaque année au rechargement de la plage située au Sud du Port de La Capte. Chaque année, sous l'impact des premières houles de tempêtes automnales, le cordon littoral disparaît quasiment. La commune souhaitait donc pérenniser cette plage en réalisant un rechargement accompagné de la mise en place d'atténuateurs de houle en géotextile (Géotubes ${ }^{\circledR}$ ), (février-mars 2008). La mise en place de ces ouvrages immergés à fortement modifié l'hydrodynamisme local, le transfert de sédiments et de manière global le système littoral de la plage de la Capte. L'étude repose sur une analyse de la dynamique morphologique (suivi topobathymétrique) de la plage avant et après la mise en place des Géotubes ${ }^{\circledR}$. La plage de la capte reste en érosion pendant les phases hivernales sans toutefois être aussi importante qu'avant. De plus, un transfert sédimentaire s'installe vers le Sud alimentant la plage Sud à partir du sable de rechargement au Nord. L'herbier de Posidonia oceanica est connu pour être un atténuateur naturel de la houle et des courants sur le fond. Une technique de cartographie de l'herbier par courantomètre profileur acoustique a donc été développée et appliquée sur ce site afin de pouvoir permettre d'évaluer l'impact global de l'herbier sur le transport sédimentaire à l'échelle du système du littoral de la Capte.

\section{Mots-clés :}

Morphologie littorale - Atténuateur de houle en géotextile - Forçages hydrodynamiques - Posidonia oceanica - Cartographie par profileur acoustique 
Thème 2 -Dynamique sédimentaire et transports des particules

\section{Introduction et contexte}

La plage de La Capte est située sur la commune d'Hyères-les-Palmiers, dans le Var (figure 1). Elle s'étend sur plus d'un kilomètre du Nord au Sud à l'Ouest du tombolo de Giens. Elle est limitée au Nord par la digue du port de La Capte.

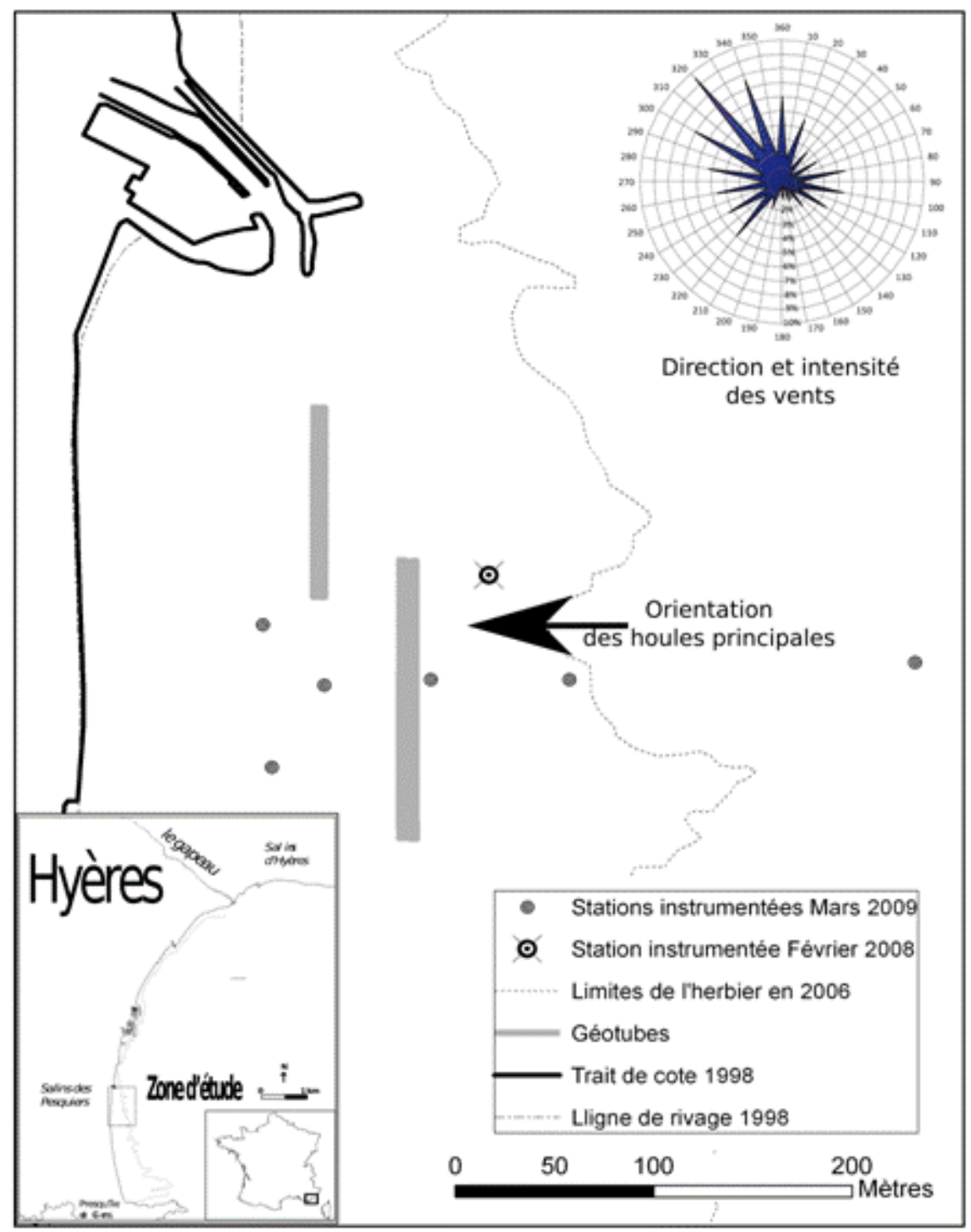

Figure 1. Localisation de la plage de la Capte, Hyères.

C'est une plage sableuse dissipative à pente faible avec un système de barres d'avant côte réduit au large duquel s'étend un herbier à Posidonia oceanica. Les digues du port bloquent les apports de la dérive littorale (orientée du Nord vers le Sud) depuis les embouchures des deux cours d'eau qui alimentent la rade en sédiments (le Gapeau et le Pansard-Maravenne). Les contraintes physiques de la rade d'Hyères (avancée du Cap Bénat et position des îles de Porquerolles et de Port-Cros) vont influencées l'orientation 
des vents dominants. Les événements tempétueux liés à la Tramontane (vents de Sud Ouest) ou au mistral (vents d'Ouest et de Nord Ouest) affectent très peu le plan d'eau dans la rade protégée par le tombolo (figure 1, rose des vents extraites de la thèse en cours de Romain Capanni). Les vents morphogènes représentent donc presque $25 \%$ des vents connus sur la zone avec une plus grande part (11.4\%) pour les vents d'Est. Ceuxci sont aussi les plus forts des vents morphogènes avec une vitesse moyenne de $5.84 \mathrm{~m} / \mathrm{s}$ et un maximum de $23 \mathrm{~m} / \mathrm{s}$. Les dynamiques perpendiculaires à la côte vont donc ici conditionner l'organisation des processus sédimentaires.

Chaque année, sous l'impact des premières houles de tempêtes automnales, le cordon littoral disparaissait quasiment. La commune souhaitait donc pérenniser cette plage en réalisant en février-mars 2008 un rechargement accompagné de la mise en place d'atténuateurs de houle en géotextile (TenCate Geotube ${ }^{\circledR}$ systems ). Les Géotubes ${ }^{\circledR}$ se composent de deux sections de longueur différentes (section nord de $100 \mathrm{~m}$; section sud de $150 \mathrm{~m}$ ). Chaque section est elle même composé de 2 boudins de géotextiles côte à côte reposant sur un tapis anti-affouillement. Les 2 sections ont été installées approximativement et respectivement à $120 \mathrm{~m}$ et $160 \mathrm{~m}$ du rivage, à une profondeur d'environ $1.70 \mathrm{~m}$ et $1.80 \mathrm{~m}$. L'objectif principal de ce travail est d'évaluer l'impact du déploiement des Géotubes ${ }^{\circledR}$ sur les processus hydrodynamiques, notamment au niveau de l'interface eau-sédiment, ainsi que sur la réponse morphodynamique (engraissement et/ou d'érosion). L'analyse se veut être quantitative et détaillée, et doit donc également prendre en compte l'effet d'atténuation sur les houles entrantes de l'herbier de $P$. Oceanica présent en zone très littorale (à partir de 3.5 mètres de fond). L'étude repose donc d'abord sur une analyse de la dynamique morphologique de la plage avant et après la mise en place des Géotubes ${ }^{\circledR}$. L'herbier de $P$. Oceanica est connu pour être un atténuateur naturel de la houle et des courants sur le fond. Une technique de cartographie de l'herbier par courantomètre profileur acoustique a donc été développée et appliquée sur ce site afin de pouvoir évaluer l'impact global de l'herbier sur le transport sédimentaire à l'échelle du système du littoral de la Capte.

\section{Méthodologie}

Des levés topographiques (plage émergée) bathymétriques (plage immergée) ont été réalisés par DGPS RTK Trimble. L'analyse spatiale des modèles numériques de terrain (MNT) extraits nous permet par différentiels de réaliser une analyse diachronique de l'évolution de la plage de la Capte. Dans le but de comprendre l'importance des forçages hydrodynamiques sur la dynamique sédimentaire de la plage de la Capte, deux phases de mesures in-situ ont été réalisées avant et après la mise en place des Géotubes ${ }^{\circledR}$. Toutefois, les résultats ne seront pas présentés dans cet article.

Alors que la littérature regorge de méthodes d'analyse de l'hydrodynamisme sur substrat sédimentaire (NIKURADSE, 1933; LI et al. ,1996), il existe très peu de travaux sur substrats végétalisés à échelle large. Pourtant, l'importance de la végétation 
sous marine sur les flux hydrologiques (NEPF et al., 1997) ou sédimentaires (GACIA \& DUARTE, 2001) a été largement démontrée. Un des objectifs de ce travail était de mettre au point une méthode de cartographie tri-dimensionnelle de la végétation sousmarine par courantomètre profileur acoustique, directement utilisable pour le développement de modèles hydrodynamiques et sédimentaires sur fonds végétalisés. WARREN \& PETERSON (2007) ont montré qu'il était possible de cartographier l'abondance de végétation sous-marine avec le même outil. Ce travail va plus loin en réalisant une bathymétrie précise des fonds sous la végétation sous-marine et en évaluant la hauteur de la canopée. Cette méthode de cartographie repose sur l'utilisation d'un profileur de courant à effet Doppler proposé par la société TRDI : l'Acoustic Doppler Current Profiler (ADCP). Le profileur acoustique est fixé sur un catamaran mis à couple de l'embarcation principale. Les levés ont réalisés le 27 octobre 2009, de la côte vers le large, au dessus de la limite d'herbier de $P$. oceanica repérée en 2007 par balisage acoustique avec un aquamètre D100 par le GIS Posidonie. Chaque mesure est géoréférencée après correction du roulis, du tangage, et de l'angle entre chaque faisceau. Une analyse spectrale spatiale est ensuite réalisée sur le signal rétro-diffusé corrigé de l'atténuation acoustique par la colonne d'eau. Les variations hautes fréquences considérées comme la partie bruitée du signal sont extraites. Les variations basses fréquences, plus importantes, correspondent à l'augmentation du signal rétro-diffusé vers le fond. Il est alors possible de calculer un coefficient $\mathrm{I}_{\mathrm{b}}$ (équation 1, unité $\mathrm{m}$.) déterminé depuis $\mathrm{m}_{0}$, le moment d'ordre 0 intégré sur les basses fréquences. Ce paramètre $\mathrm{Ib}$ permet in-fine d'automatiser l'identification de la typologie de fond. Lorsqu'il est inférieur à $20 \mathrm{~m}$, cela indique alors la présence d'un herbier de $P$. oceanica. $\mathrm{I}_{\mathrm{b}}=4 \times \mathrm{m}_{0}$

Une cartographie assez précise peut donc résulter de ce type de données puisque pour chaque faisceau, on peut définir la profondeur, le type de fond, la hauteur de végétation et la position géographique. Les marges d'erreurs associées à nos mesures prennent en compte, la largeur du transducteur du profileur acoustique $(86.4 \mathrm{~mm})$ et l'ouverture autour du faisceau acoustique émis $\left(15^{\circ}\right)$, et la superposition de points de mesures. On peut ainsi estimer la précision du positionnement horizontal à $80 \mathrm{~cm}$.

\section{Résultats}

Les mesures réalisées lors des phases d'instrumentation immergées (avant et après mise en place des Géotubes ${ }^{\circledR}$ nous ont permis d'enregistrer des régimes de tempêtes avec des houles dont les hauteurs significatives atteignaient un mètre et des périodes comprises entre 5 et 7 secondes suivant l'intensité des vents locaux. De part le contexte géomorphologique de la plage, l'orientation des houles sont toutes quasi-normales à la côte. Ces régimes de houles pour ce type de plage sableuse sont donc relativement conséquent morphologiquement. 


\section{XI $I^{\text {èmes }}$ Journées Nationales Génie Côtier - Génie Civil}

Les Sables d'Olonne, 22-25 juin 2010

Avant la mise en place des Géotubes ${ }^{\circledR}$ en mars-avril 2008, l'étude du fonctionnement de la plage montrait une érosion importante de la plage de la Capte lors des périodes hivernales (étude réalisée entre novembre 2007 et février 2008). Le recul du trait de côte atteignait en certains endroits le pied de la digue frontale réalisant un déchaussement de plus de $30 \mathrm{~cm}$ localement (figure 2). A l'entrée du port, nous avions toutefois constaté une zone en colmatage avec une accrétion atteignant 70 à $90 \mathrm{~cm}$. Sur la figure 2, les traits de côte de février 2008, juillet 2008 et mars 2009 sont surimposé au MNT de mars 2009. Une avancée considérable de plus de $30 \mathrm{~m}$ de la plage est constatée entre février et juillet 2008. Par la suite entre juillet 2008 et mars 2009, il est observé un recul de plus de $20 \mathrm{~m}$ de la plage dans la partie nord.

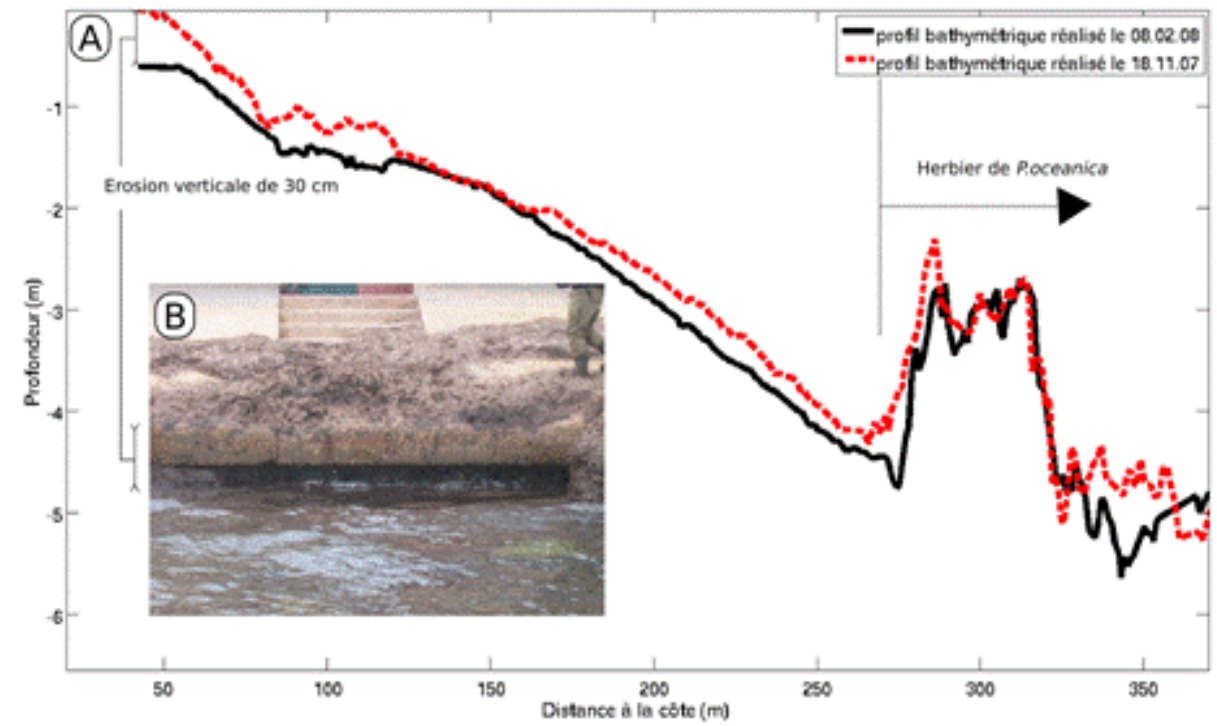

Figure 2. (A) Évolution des profils bathymétriques réalisés les 18 novembre 2007 (rouge) et 08 février 2008. (B) Photographie prise le 8 février 2008 représentant l'important déchaussement de la digue de fond de plage sur la plage de la Capte.

L'analyse d'autres levés topographiques montrent que dans la partie sud, il y a une évolution positive du trait de côte depuis la mise en place des Géotubes ${ }^{\circledR}$. La figure 3B présente le différentiel des MNT de février 2008 et juillet 2008. Une nette accrétion de l'ensemble de la plage depuis le haut de plage (avec plus de $1.5 \mathrm{~m}$ de sable) jusqu'à $2 \mathrm{~m}$ de profondeur est observée. Ceci correspond au $12000 \mathrm{~m}^{3}$ de sable de rechargement sur la plage émergée qui accompagnait la mise en place des atténuateurs de houle. On observe également une zone importante d'érosion au Sud-Est du port. La délimitation de cette zone correspond à l'espace de dragage des sédiments destinées à alimenter les tubes de géotextiles. La figure 3C présente les différentiels des MNT de juillet 2008 et mars 2009. Cette période couvre une série importante d'événement de tempêtes d'Est notamment en décembre 2008. 


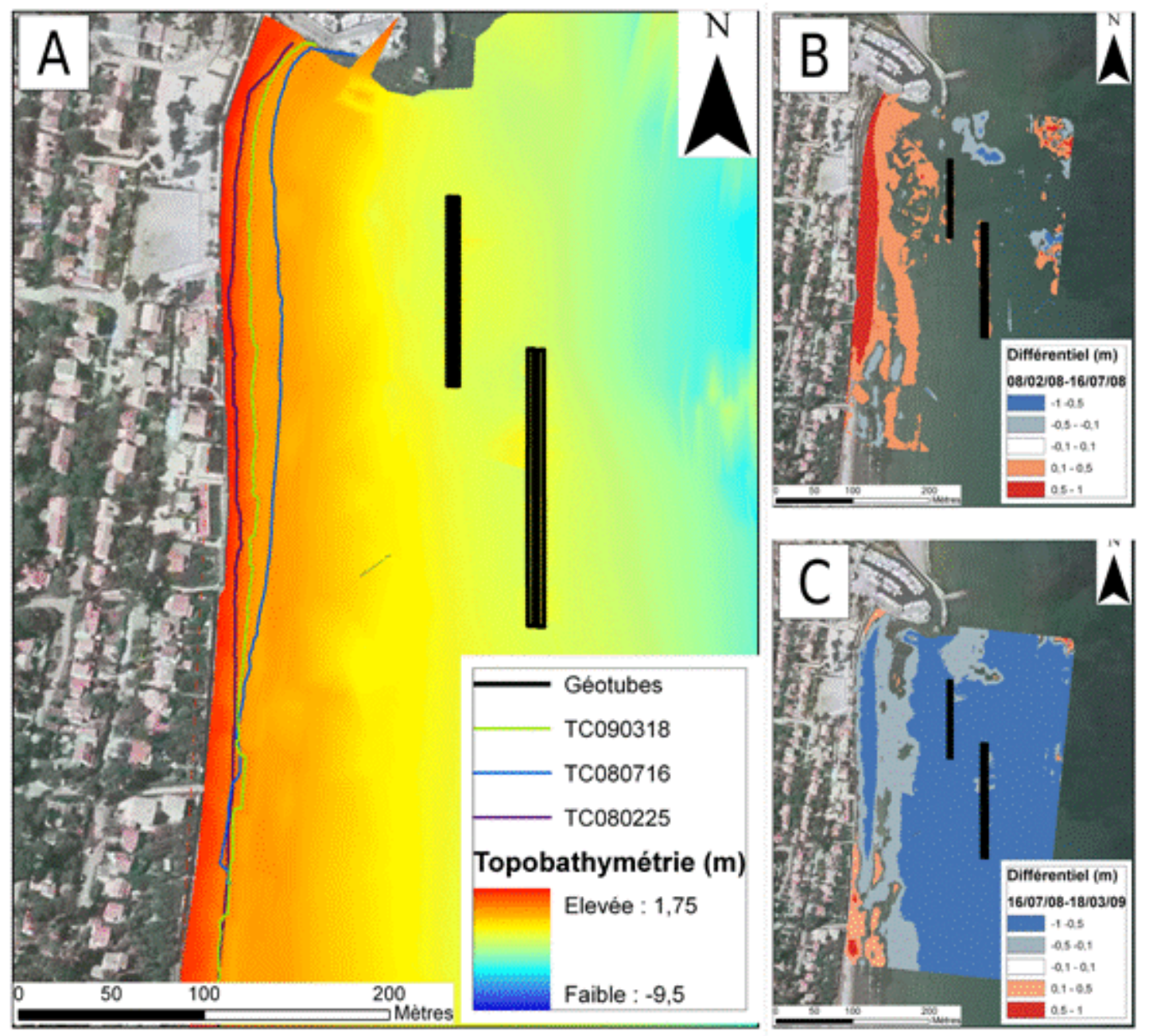

Figure 3. (A) MNT réalisé en mars 2009 et évolution des traits de côte (février et juillet 2008, mars 2009). (B) Différentiel des MNT entre février et juillet 2008. (C) Différentiel des MNT entre juillet 2008 et mars 2009.

Nous pouvons observer une érosion importante généralisée dans la partie nord de la plage. Toutefois au sud des Géotubes ${ }^{\circledR}$, une accrétion est constatée pour la partie supérieure de la plage, de $-60 \mathrm{~cm}$ à $+1.50 \mathrm{~m}$. L'analyse du fonctionnement estival après la mise en place des Géotubes ${ }^{\circledR}$ indique une stabilité du trait de côte. Une accumulation dans la zone de rive semble s'opposer au déficit sédimentaire observé dans la partie supérieure de la plage aérienne. Le transfert sédimentaire nord sud précédemment décrit pendant la période hivernale reste très limité.

L'un des objectifs de ce travail était de réaliser une méthodologie de cartographie des environnements benthiques, et plus particulièrement de pouvoir distinguer la présence de végétation sous-marine. La figure 4 présente le résultat de la cartographie de l'herbier de $P$. oceanica réalisée à partir d'un profileur acoustique. La limite supérieure de l'herbier réalisée par profileur acoustique suit de manière extrêmement satisfaisante la 
limite de l'herbier réalisée par le GIS Posidonie en 2006 (compilation de données de terrain et de travaux d'identification sur ortho-photographie). Nous pouvons également observer une très bonne correspondance avec les limites aquamètre D100 sud et centrale. Les successions du nord au sud d'avancée et recul de la limite haute de l'herbier sont ainsi bien représentées par notre méthodologie. Au niveau de la limite aquamètre nord, il $\mathrm{y}$ a une différence marquée entre les 3 méthodologies. Nous constatons ainsi une limite haute de l'herbier en retrait de $10 \mathrm{~m}$ entre 2006 et 2007 et d'un peu plus de $10 \mathrm{~m}$ entre 2007 et 2009.

Pourtant d'après le suivi actuel réalisé par le GIS Posidonie (GRATIOT, 2009), aucun recul réel n'a été observé, bien que de nombreuses mattes mortes aient été identifiées. Enfin, à l'intérieur de la zone cartographiée, de nombreuses zones semblent indiquée la présence de sable. Il y a tout d'abord une attention particulière à porter sur la méthodologie de la représentation de cette carte. En effet, une interpolation spatiale de type triangulation Delaunay a été réalisée et induit des exagérations dans les zones ou la fréquence spatiales d'échantillonnage est peu dense. Toutefois, d'après nos propres observations, ainsi que celles du GIS Posidonie, il existe au sein de l'herbier de nombreuses disparités, induites par la topographie sous-jacente et par l'évolution de l'herbier en zone de mattes mortes. Il est ainsi possible de retrouver des zones de fortes accumulations sédimentaires que notre méthodologie identifie. Les hauteurs de canopée ont été extraites depuis l'analyse de chacun des signaux rétro-diffusés. La hauteur de canopée est très variable et des valeurs entre $25 \mathrm{~cm}$ et $98 \mathrm{~cm}$ sont ainsi déterminées. Cette fluctuation est liée à l'état de l'herbier, puisque des herbiers juvéniles ou dégradés n'auront pas la même hauteur. Toutefois, la moyenne de la canopée réalisée sur quelques levés donne des valeurs de $55 \mathrm{~cm}$ identique à la hauteur moyenne de $55 \mathrm{~cm}$ relevé par BOUDOURESQUE et al. (2006).

\section{Conclusion}

La mise en place des Géotubes ${ }^{\circledR}$ à modifié le fonctionnement hydrodynamique de la plage de la Capte dont nous constatons dans cette étude les impacts géomorphologiques. Malgré des forçages marins importants pendant l'hiver 2008-2009, le trait de côte de 2009 montre une avancée d'une dizaine de mètres par rapport à l'état initial constaté en 2007. Cette avancée, dans la partie nord de la plage, est le résultat du rechargement sédimentaire important de 2008 associé à une phase hivernale érosive limitée. Un transfert sédimentaire vers le sud s'est mis en place dans la zone de jet de rive permettant l'accrétion de la plage au sud. Les Géotubes ${ }^{\circledR}$ semblent donc avoir limité la perte sédimentaire de la plage par rapport au fonctionnement observé avant leur mise en place. Cette perte sédimentaire pourrait toutefois avoir des conséquences à plus longterme. En effet à l'ouest des atténuateurs de houle, la zone touchée par l'érosion met progressivement à nu un substrat de type beach rock (identifié précédemment lors d'une campagne sismique). 
Thème 2 - Dynamique sédimentaire et transports des particules

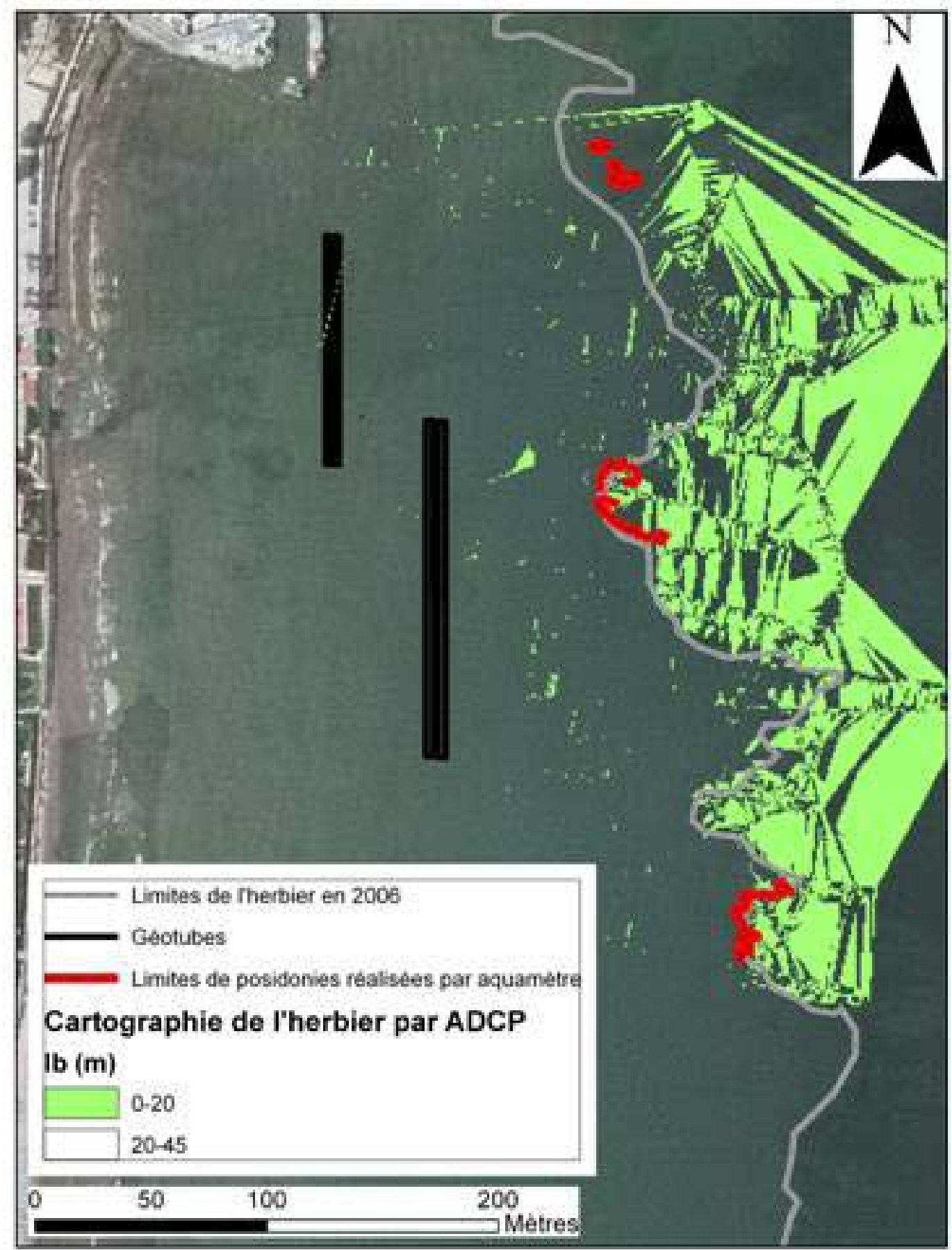

Figure 4. Cartographie de l'herbier par profileur acoustique.

Dans l'herbier de $P$. oceanica, la dynamique sédimentaire est complexe avec des zones alternant érosion et dépôt. Il était donc important de réaliser une cartographie de la végétation sous-marine afin in-fine d'améliorer l'état de nos connaissances sur l'impact de la végétation sous-marine sur le système sédimentaire en milieu côtier. La méthode développée à travers cette étude permet une cartographie précise de la végétation sous 
marine en termes de présence, de profondeur d'implantation et de hauteur d'herbier. Toutefois il semble nécessaire de développé une typologie plus poussée de la morphologie de chaque état de l'herbier couplé éventuellement à une reconnaissance du fond par plongée afin de définir si la hauteur d'herbier peut correspondre à un état dégradé, juvénile ou mature de l'herbier de $P$. oceanica. Par la suite, la connaissance de l'ensemble de ces caractéristiques permettra d'intégrer l'interaction entre l'hydrodynamisme et la canopée d'une végétation immergée en milieu côtier. Ainsi, les travaux de modélisation de CHEN et al. (2007) s'appuie sur le calcul d'un coefficient de friction, fonction de la rugosité et dépendant de la densité de faisceaux par $\mathrm{m}^{2}$, la largeur de feuilles (en moyenne égale à un centimètre sur l'herbier de $P$. oceanica), la hauteur de canopée et la profondeur d'eau. L'ensemble de ces paramètres peuvent être désormais extrait de la méthodologie développée.

\section{Remerciements}

Cette étude est financée à travers deux programmes :

- MICROLIT RELIEFS INSU/SHOM piloté par le SHOM et l'Université de Perpignan - IMAHGEO financé par la municipalité de Hyères.

Sur les figures 2 et 3 , les données de cette étude sont surimposées à une orthophotographie $\mathrm{IGN}^{\circledR} 2003$.

\section{Références bibliographiques}

BOUDOURESQUE C., BERNARD G., BONHOMME P., CHARBONNEL E., DIVIACCIO G. (2006). Préservation et conservation des herbiers à Posidonia oceanica. Ramoge pub., $200 \mathrm{p}$.

CHEN S., SANFORD L.P., KOCH E.W., SHI F., NORTH E.W. (2007). A nearshore model to investigate the effects of seagrass bed geometry on wave attenuation and suspended sediment transport. Estuaries and Coasts, vol. 30, pp 296-310.

GACIA E., DUARTE C.M. (2001). Sediment Retention by a Mediterranean Posidonia oceanica Meadow: The Balance between Deposition and Resuspension. Estuarine Coastal and Shelf Science, vol. 52, pp 505-514. doi:10.1006/ecss.2000.0753

GRATIOT J. (2009). Rapport GIS Posidonie La Capte.

LI M., WRIGHT L., AMOS C. (1996). Predicting ripple roughness and sand resuspension under combined flows in a shoreface environment. Marine Geology, vol. 130, pp 139-161. doi:10.1016/0025-3227(95)00132-8

NEPF H.M., MUGNIER C.G., ZAVISTOSKI R.A. (1997). The Effects of Vegetation on Longitudinal Dispersion. Estuarine, Coastal and Shelf Science, vol. 44, pp 675-684. doi:10.1006/ecss.1996.0169

NIKURADSE J. (1933). Strömungsgesetze in Rauhen Rohren. Forschung arb. ing. wesen, vol. 36 . 
Thème 2 -Dynamique sédimentaire et transports des particules

WARREN J.D., PETERSON B.J. (2007). Use of a 600-kHz Acoustic Doppler Current Profiler to measure estuarine bottom type, relative abundance of submerged aquatic vegetation, and eelgrass canopy height. Estuarine Coastal and Shelf Science, vol.72, pp 53-62. doi:10.1016/j.ecss.2006.10.026 